\title{
MEDIDAS DE MORBIDADE PRODUZIDAS POR DUAS FONTES DIVERSAS. RIBEIRÃO PRETO, SP (BRASIL), 1975 *
}

Carvalheiro, J. Da R. \& CaRvalheiro, C. D. G. Medidas de morbidade produzidas por duas fontes diversas. Ribeirão Preto, SP (Brasil), 1975. Rev. Saúde públ., S. Paulo, 13:265-70, 1979.

Resumo: Foram estudadas duas fontes de dados de morbidade: levantamento por entrevistas domiciliárias que corresponde a uma amostra representativa da cidade de Ribeirão Preto, e a morbidade em egressos hospitalares, produzida pelo Centro de Processamento de Dados Hospitalares do Departamento de Medicina Social da Faculdade de Medicina de Ribeirão Preto, USP, em 1975. Foram destacados 10 Grupos de Doenças da Classificação Internacional de Doenças (CID), Revisão de 1965. Em 1975, o número de hospitalizações de residentes foi de 1,1 por cem pessoas-mês. A morbidade proporcional dos vários grupos de doenças analisados mostrou, em ordem decrescente de freqüência nos egressos hospitalares, os Grupos da CID; VIII, VII, XVI, XVII, IX,X,I, XIII e $V$ mais $V I$, sendo que os demais apresentam-se em pequena proporção. As entrevistas, mostraram: Grupos VIII, IX,V mais VI, XVI, XIII, VII, I, X, XVII $e$ os demais em pequeno número. A ordenação dos diferentes grupos não é a mesma quando o diagnóstico é hospitalar ou resultante de entrevista domiciliária. A aplicação de um modelo ecológico clássico de atenção médica mostrou que, mensalmente, 608 pessoas adoecem, das quais apenas 11 säo hospitalizadas.

Unitermos: Morbidade. Morbidade hospitalar.

As medidas de frequiência das doenças apresentam uma série de problemas, pois provêm de diversas fontes de registro, que usam metodologia variada, o que dificulta a análise do quadro geral da morbidade. Muitas vezes, ainda, diferentes fontes atuam de modo complementar no diagnóstico da morbidade. Entretanto, desde que se leve sempre em conta as diferenças em tempo, lugar e critérios, estas medidas dão-nos idéia de alguns aspectos da história natural da atenção médica ao paciente.

O objetivo do presente trabalho foi o de obter medidas de morbidade de duas fontes de dados: um levantamento por entrevistas domiciliárias e de um Centro de Processa-

* Apresentado na 30 Reunião Anual da Sociedade Brasileira para o Progresso da Ciência, Săo Paulo, 1978.

** Do Departamento de Medicina Social da Faculdade de Medicina de Ribeirão Preto - 14100 - Ribeirão Preto, SP - Brasil. 
CARVAlHeiro, J. da R. \& CARVAlheiro, C D. G. Medidas de morbidade produzidas por duas fontes diversas. Ribeirão Preto, SP (Brasil), 1975. Rer, Saúde públ., S. Paulo, 13: 265-70. 1979 .

mento de Dados Hospitalares, para se analisar alguns aspectos ligados à atenção médica.

\section{MATERIAL E MIETODOS}

Os dados estudados procederam das se. guintes fontes:

1. Levantamento por entrevisias domiciliárias, sobre condiçōes de saúde. Este levantamento, rinculado à Faculdade de Medicina de Ribeirão Preto da USP, foi iniciado em 1972, atingindo, no segundo semestre de 1974, toda a zona urbana da cidade?. Corresponde a uma amostra representativa da população da cidade de Ribeirão Preto, Preto, retirada de um painel de 5.200 domicílios. Semanalmente sãc visitadas 380 famílias, correspondendo a $1 / 100$ do total de famílias residentes em 1970, segundo o censo de 1970.

A presente investigação abrangeu 25 semanas epidemiológicas de 1975 . Nestas 25 semanas, entrevistadoras treinadas investigaram a ocorrência de episódios de morbidade em 8.512 famílias, correspondendo a 38.274 membros do núcleo familiar. A morbidade foi codificada segundo os 17 grupos da Classificação Internacional de Donenças -- Revisão de 1965:.

2. Centro de Processamento de Dados Hospitalares (CPDH) do Departamento de Medicina Social da Faculdade de Medicina de Ribeirão Preto da USP. Funcionando desde 1970, o CPDH recebe as folhas de alta hospitalar preenchidas pelos hospitais gerais e Pronto Socorro Infantil da cidade, coleta e codifica as informações, transferindo-as para cartões IBM. Os dados são processados eletronicamente e publicaçoes trimestrais sāo preparadas e distribuidas às instituições que mantêm convênio com o CPDH i...

Os dados do presente trabalho corresponden a 32.645 internaçōes, de residentes em Ribeirāo Preto, realizadas nos hospitais gerais e Pronto Socorro Infantil, em 1975. Essas internações foram classificadas pelo diagnósticn principal, segundo os 17 grupos da CID \%.

\section{RESULTADOS E DISCUSSĀO}

Nas semanas epidemiológicas analisadas, a morbidade geral referida foi de 11.637 episódios, o que equivale a 30,4 episódios de doença por cem pessoas por quinzena oll 60,8 episódios de doença por cem pessoas por mês. A Tabela 1 mostra-nos estes valores, bem como a variação da morbidade geral referida no decorrer das 25 semanas. Observa-se que há pequenas oscilaçōes entre a 14a. e a 29a. semana, e daí em diante, até a 49a. semana, verificase um decréscimo da morbidade geral referida, que chegou próximo a $22 \%$ nas últimas semanas. É difícil interpretar este decréscimo, não sendo possivel afastar a hipótese de artefato, motivado pelo progressivo treinamento do pessoal de trabalho de campo.

Se separarmos as 10 causas mais frequientes de morbidade referida, verificamos que 25,4\% corresponderam ao Grupo VIII Doenças do Aparelho Respiratório, conforme observamos na Tabela 2. Vem a seguir em ordem de freqüência, $15,8 \%$ para o Grupo IX - Doenças do Aparelho Digestivo, $12,5 \%$ para os Grupos V mais VI Transtornos Mentais e Doenças do Sistema Nervoso e dos Orgãos dos Sentidos, 11,0\% para o Grupo XVI - Sintomas e Estados Mórbidos Mal Definidos, $10,3 \%$ para o Grupo XIII - Doenças do Sistema Muscular e Tecido Conjuntivo, 9,0\% para 0 Grupo VIl - Doenças do Aparelho Circulatório, 5,2\% para o Grupo 1 - Doenças Infecciosas, $4,2 \%$ para o Grupo X Doenças do Aparelho Geniturinário e $2,0 \%$ para o Grupo XVII - Acidentes. Envenenamentos e Violencias. 
CARVAlHeiro, J. da R. \& CARVAlHeiro, C. D. G. Medidas de morbidade produzidas por duas fontes diversas. Ribeirão Preto, SP (Brasil), 1975. Rev. Saúde públ., S. Paulo, 13: 265-70, 1979.

T A B ELA 1

Familias entrevistadas, número de membros e morbidade geral referida, segundo a semana epidemiológica, Ribeirão Preto, 1975.

\begin{tabular}{|c|c|c|c|c|}
\hline \multirow{2}{*}{ Semana } & \multirow{2}{*}{ No de famílias } & \multirow{2}{*}{$\begin{array}{l}\text { No de membros } \\
\text { núcleo familiar }\end{array}$} & \multicolumn{2}{|c|}{ Morbidade geral referida } \\
\hline & & & Númem & Coeficiente $(\%)$ \\
\hline 14 & 354 & 1597 & 621 & 38,9 \\
\hline 15 & 346 & 1534 & 549 & 35.8 \\
\hline 16 & 348 & 1528 & 525 & 34.4 \\
\hline 17 & 343 & 1542 & 494 & 32.0 \\
\hline 18 & 346 & 1561 & 478 & 30,6 \\
\hline 19 & 349 & 1553 & 457 & 29,4 \\
\hline 20 & 351 & 1566 & 578 & 36,9 \\
\hline 21 & 348 & 1563 & 550 & 35,2 \\
\hline 22 & 330 & 1502 & 422 & 28,1 \\
\hline 23 & 342 & 1558 & 544 & 34,9 \\
\hline 24 & 343 & 1610 & 536 & 33.3 \\
\hline 25 & 338 & 1570 & 474 & 30,2 \\
\hline 26 & 342 & 1595 & 539 & 33,8 \\
\hline 27 & 327 & 1480 & 485 & 32,8 \\
\hline 29 & 320 & 1402 & 426 & 30,4 \\
\hline 81 & 304 & 1357 & 394 & 29.0 \\
\hline 33 & 345 & 1550 & 433 & 27,9 \\
\hline 35 & 332 & 1474 & 379 & 26,4 \\
\hline 37 & 341 & 154 & 431 & 27.9 \\
\hline 39 & 345 & 1528 & 451 & 29,5 \\
\hline 41 & 344 & 1558 & 379 & 24,8 \\
\hline 43 & 343 & 1543 & 415 & 26,9 \\
\hline 45 & 341 & 1510 & 395 & 26,2 \\
\hline 47 & 344 & 1558 & 349 & 22,4 \\
\hline 49 & 345 & 1492 & 333 & 22.3 \\
\hline Total & 8512 & 38274 & 11637 & 30,4 \\
\hline
\end{tabular}

A Tabela 3 mostra-nos que, quanto às internações realizadas nos hospitais, $15,2 \%$ são por Doenças do Aparelho Respiratório (Grupo VIII), 11,4\% por Doenças do Aparelho Circulatório (Grupo VII), 9,2\% são do Grupo XVI - Sintomas e Estados Mórbidos Mal Definidos, $8,1 \%$ por Acidentes, Envenenamentos e Violências (Grupo XVII), 6,4\% por Doenças do Aparelho Digestivo (Grupo IX) e também Doenças do Aparelho Geniturinário (Grupo X), $3,5 \%$ por Doenças Infecciosas e Parasitárias (Grupo 1), 3,3\% por Doenças do Sistema Osteomuscular e Conjuntivo (Grupo XIII), e 3,0\% por Transtornos Mentais e Doenças do Sistema Nervoso e Órgãos dos Sentidos
(Grupos V mais VI). O coeficiente de hospitalização foi de $13,19 \%$, o que corresponde a 1,1 hospitalizaçōes por cem pessoas por mês. Os resultados mostram que a ordenação dos diferentes grupos não é a mesma quando o diagnóstico é hospitalar ou resultante de entrevista domiciliária (coeficiente de concordância de Kendall. $\mathrm{W}=0,5833,8$ G.L., $0,30<\mathrm{p}<0,50)$. Comparando-se as Tabelas 2 e 3 , verificamos que o Grupo VIII - Doenças do Aparelho Respiratório, é a causa mais freqüente tanto de morbidade referida, como de hospitalizaçōes, O grupo $\mathrm{V}$ mais $\mathrm{VI}-$ Transtornos Mentais e Doenças do Sistema Nervoso e órgãos dos Sentidos é a $3^{a}$ 
CARVALHEIRO, J. da R. \& CARVALHEIRO, C D. G. Medidas de morbidade produzidas por duas fontes diversas. Ribeirão Preto, SP (Brasil), 1975. Rev. Saúde públ., S. Paulo, 13: 265-70, 1979.

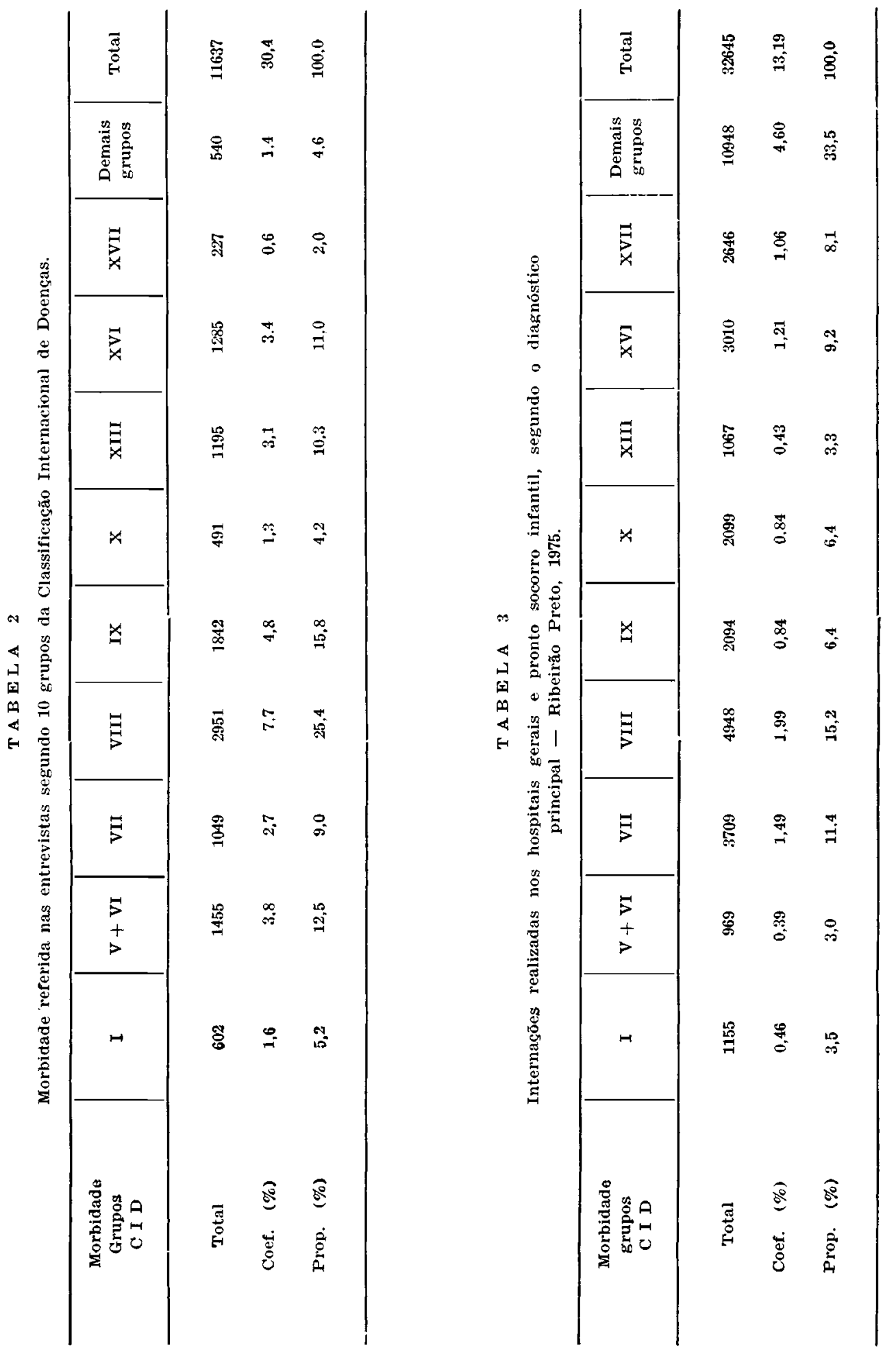


CARVAlHEIRo, J. da R. \& CARVAlheiro, C D. G. Medidas de morbidade produzidas por duas fontes diversas. Ribeirão Preto, SP (Brasil), 1975. Rev. Saúde públ., S. Paulo, 13: 265-70, 1979.

causa mais frequiente na morbidade referida, passando para o último lugar nas hospitalizações. O Grupo XVII, Acidentes, Envenenamentos e Violências, que ocupa o último lugar entre a morbidade referida, passa para o 4o lugar nas hospitalizações.

Chama-nos ainda a atenção que o Grupo XVI - Sintomas e Estados Mórbidos Mal Definidos, que ocupa o 40 lugar na morbidade referida (o que se poderia explicar por uma série de dificuldades inerentes à técnica de entrevista, ao entrevistador ou ao paciente), atinge o 3 \% lugar entre as hospitalizações, mostrando a precariedade da quantidade e/ou qualidade da atenção médica.

Os valores encontrados para o coeficiente de morbidade referida $(60,8$ episódios de doença por cem pessoas/por mês) e o coeficiente de hospitalização (1,1 hospitalizações por cem pessoas por mês) são diferentes dos valores citados por White $e$ col. para a Grã-Bretanha e Estados Unidos. Segundo esses autores, de cada mil adultos na população, 750 reconhecem um episódio de doença ou lesão e, destes, 9 são hospitalizados.

Considerando que os 60,8 episódios de doença por cem pessoas por mês podem corresponder a mais de um episódio por pessoa, o que superestima este dado, vemos que a morbidade referida por White e col.* ainda assim é maior do que a nossa. Se considerarmos a variação demográfica existente nas composições etárias da população britânica ou americana e da nossa, ou seja, que populaçōes com maior percentual de velhos devem ter maior morbidade que populações jovens, realmente esperariamos que este fato ocorresse. Além disso, deve influir também, o padrão cultural da população, o nivel educacional e sócio-econômico, além de variáveis ligadas à estrutura dos serviços de saúde.

Quanto ao coeficiente de hospitalizaçāo encontrado ( 1,1 hospitalizações por cem pessoas por mês), ele é um pouco mais alto do que vários dos citados por White e col.4, que oscilam em torno de 0,7 hospitalizaçōes por cem pessoas adultas por mês. Isto poderia ocorrer em parte, pelo excesso relativo de oferta de atenção médica existente no Municipio e em parte pela maior gravidade das doenças no nosso meio. Poderia, finalmente, ocorrer um diferencial segundo a fonte de pagamento: previdência, particular e outras. Nas 25 semanas epidemiológicas de 1975, a fonte de pagamento para consulta médica foi o INPS em $42,8 \%$, gratuita em $28,1 \%$ e particular em $16,4 \%$, estando os $12,7 \%$ restantes distribuídos entre outras fontes.

\section{CONCLUSÕES}

1. O coeficiente de morbidade referida pela população foi de 60,8 episódios de doença por cem pessoas por mês, para Ribeirão Preto, 1975.

2. O coeficiente de hospitalização foi de 1,1 hospitalizaçōes por cem pessoas por mês.

3. Um confronto dos diagnósticos da morbidade hospitalar com aqueles da morbidade referida nas entrevistas domiciliárias revela uma discrepância que não pode ser imputada ao acaso. Além da diferença entre os métodos diagnósticos (a morbidade hospitalar é uma definição técnica enquanto a outra deriva de uma informação leiga) é platusivel admitir que haja uma composição real da morbidade ao nível da população que, triada diferencialmente, gera uma demanda hospitalar de composição distinta da original.

4. As diferenças destes coeficientes com os valores citados por White e col.4 devem-se, provavelmente, a numerosos fatores relacionados não só com a definição dos coeficientes, como diferenças nos padrões culturais das populações, nivel educacional e sócio-econômico, na distribuição etária das populações, variação na oferta de atenção médica e diferenças na gravidade das doenças $e$ quanto à fonte de pagamento. 
CARVAlHeiro, J. da R. \& CARVAlheiro, C. D. G. Medidas de morbidade produzidas por duas fontes diversas. Ribeirão Preto, SP (Brasil). 1975. Rev. Saúde públ., S. Paulo, 13: 265-70, 1979.

Carvalheiro, J. Da R. \& Carvalheiro, C. D. G. IMorbidity statistics furnished by two different sources. Ribeiräo Preto, SP (Brazil), 1975/ Rev. Saúde públ., S. Paulo, $13: 265-70,1979$.

ABSTRACT: Morbidity statistics from two different sources are the subject of this report. The sources were a home interview survey of a representative sampling in the city of Ribeirão Preto and hospital in patient statistics furnished by the Hospital Data Processing Center of the Department of Social Medicine of the School of Medicine of Ribeirão Preto (University of S. Paulo) in 1975. Ten groups of diseases in the International Classification of Diseases (ICD), 1965 Revision, were focused on. The number of hospitalizations of city residents was 1.1 per 100 persons per month in 1975 . Among hospital in patients, the incidence of morbidity of the ICD Groups analyzed was, in a decreasing order of frequency, as follows: VIII, VII, XVI, XVII, IX, X,I,XIII, and V plus VI. The other Groups are represented to a lesser degree. In the home interview survey, morbidity showed up in these ICD Groups: VIII, IX, V plus VI, XVI, XIII, $V I I, I, X$, and $X V I I$, with the other Groups presenting a lower rate of incidence. Clearly the order of the different groups is not the same when the diagnosis of morbidity is taken from hospital data files and from home interviews. The application of the classical ecological model of medical care shows that 608 persons feel sick per month, and, of these, only 11 are hospitalized.

UNITERMS: Morbidity. Hospital morbidity.

\section{REFERENCIAS BIBLIOGRAFICAS}

1. AZEVEDO BARROS. M. B. Estudo da morbidade hospitalar no Municipio de Ribeirão Preto, em 1975. Ribeirão Preto, 1977. [Dissertação de Mestrado - Faculdade de Medicina de Ribeirão Preto da USP'].

2. CARVALHEIRO, J. R. Levantamento de condicozes de saúde por entrevistas domiciliarias. Ribeirão Preto, 1975. [Tese de Livre-Docência - Faculdade de Medicina de Ribeirão Preto da USP].

3. ORGANIZAÇAO PANAMERICANA DA SAÚ$\mathrm{DE}$. Manual da classificação estatística internacional de doenças, lesoes e causas de bbitos; revisão 1.965. Washington, DC. 1969. v. 1.
4. WHITE. K. L. et al. The echlogy of medical care. New Engl. J. Med., 265:885-92. 1961.

5. YAZLLE ROCHA, J. S. Estudo da utilizacado de leitos hospitalares gerais do municipio de Ribeirão Preto, pela população do Municipio no ano de 1972. Ribeirão Preto, 1974. [Tese de Doutoramento - Faculdade de Medicina de Ribeirão Preto da USP].

Recebido para publicacão em 22/03/1979 Aprovado para publicaçāo em 19/06/1979 